PROCEEDINGS OF THE

AMERICAN MATHEMATICAL SOCIETY

Volume 132, Number 8, Pages 2291-2302

S 0002-9939(04)07319-8

Article electronically published on March 25, 2004

\title{
BACKWARD EXTENSIONS OF SUBNORMAL OPERATORS
}

\author{
IL BONG JUNG, ALAN LAMBERT, AND JAN STOCHEL
}

(Communicated by Joseph A. Ball)

\begin{abstract}
The concept of backward extension for subnormal weighted shifts is generalized to arbitrary subnormal operators. Several differences and similarities in these contexts are explored, with emphasis on the structure of the underlying measures.
\end{abstract}

\section{INTRODUCTION}

An operator $T$ on a Hilbert space $\mathcal{H}$ is subnormal if there is a normal operator $N$ on a Hilbert space containing $\mathcal{H}$ as a closed subspace such that the restriction of $N$ to $\mathcal{H}$ is $T$. Weighted shifts have provided many examples of subnormal operators and motivation for analysis of this family of operators. In particular, weighted shifts most directly show the intimate relationships between shifts and moment sequences (cf. [12]). A specific area of investigation which has proven to be quite productive is that of backward extensions. Given an orthonormal basis $\left\{e_{0}, e_{1}, \ldots\right\}$ for $\mathcal{H}$ and a subnormal weighted shift $W$ with weight sequence $\alpha=\left\{\alpha_{n}\right\}_{n=0}^{\infty}$ in $\mathbb{R}^{+}$ given by $W e_{n}=\alpha_{n} e_{n+1}$, R. Curto described conditions on the measure and moment sequence associated with $W$ that guarantee that there is a shift with weights $\left\{x, \alpha_{0}, \alpha_{1}, \ldots\right\}$ that is also subnormal. The full description of Curto's theorem is presented later in the article. Further results followed this direction of investigation concerning the gap between subnormal and hyponormal operators (cf. [3], [6], 7] and [8]).

There is a description of subnormality for an abstract operator $T$ in terms of weighted shifts; namely, $T$ is injective subnormal if and only if for each $h \neq 0$ in $\mathcal{H}$, the weighted shift with weight sequence $\left\{\left\|T^{n+1} h\right\| /\left\|T^{n} h\right\|\right\}$ is subnormal ([9]). Our main goal in this note is to use Curto's theorem to develop general conditions analogous to the one-dimensional extensions (one-step backward extensions) central to Curto's theorem and its corollaries. To this end, we explore the two abstract measure-theoretic descriptions of subnormality for a cyclic operator $T$ of norm one:

(i) $T$ is subnormal if and only if there is a finite Borel measure $\nu$ on the closed unit disk $\mathbb{D}$ such that $T$ is unitarily equivalent to the operator $S_{\nu}$ of multiplication by the independent variable $z$ on $H^{2}(\nu)$, the $L^{2}(\nu)$-closure of the set of all polynomials on $\mathbb{D}$.

(ii) For $T$ injective, $T$ is subnormal if and only if for each $h$ in $\mathcal{H}$ with $\|h\|=1$, there is a probability measure $\mu_{h}$ such that for every $n \geq 0,\left\|T^{n} h\right\|^{2}=\int_{0}^{1} t^{n} d \mu_{h}(t)$.

Received by the editors July 31, 2001 and, in revised form, April 15, 2003.

2000 Mathematics Subject Classification. Primary 47B20; Secondary 47B37, 44A60.

Key words and phrases. Cyclic vectors, subnormal operators, moment measures. 
We will show that there are substantial similarities and differences in backward extension theory for shifts and general subnormal operators. Specifically, each subnormal weighted shift itself gives rise to a large family of subnormal shifts via the moment sequence technique alluded to earlier. It was shown in [5] that if the primary shift allows backward extension, then so do all these secondary shifts, and indeed, there are absolute continuity relationships among the associated measures. This general backward extension phenomenon will be referred to as full backward extensions. A major focus of our interest is characterizing those subnormal operators that allow full backward extension. The automatic fullness of backward extension enjoyed by weighted shifts is not automatic for arbitrary subnormal operators. The difference is shown to be intimately related to the various measures involved. We then investigate multiple-step backward (and full backward) extensions. Even in the weighted shift case, one-step extension does not guarantee further extension; this was a core consideration in [5].

Our analysis of full backward extension focuses on the (potential) differences between the operators of multiplication by $z$ on $L^{2}(\nu)$ and on $R^{2}(\nu)$, where $R^{2}(\nu)$ is the $L^{2}(\nu)$-closure of the set of Laurent polynomials (contingent of course on having $\left.\left\{z^{-k}: k=1,2, \ldots\right\} \subset L^{2}(\nu)\right)$.

\section{Subnormal operators - The general Case}

Throughout this section $T$ is assumed to be a bounded subnormal operator on a (complex) Hilbert space $\mathcal{H}$. It is known (cf. 9]; see also [13. Prop. 2.3]) that for each nonzero vector $h \in \mathcal{H}$, there exists a unique positive Borel measure $\mu_{h}$ on $[0, \infty)$ such that

$$
\left\|T^{n} h\right\|^{2}=\|h\|^{2} \int t^{n} d \mu_{h}(t), \quad n \geq 0
$$

The measure $\mu_{h}$ is probabilistic and its closed support is contained in $\left[0,\|T\|^{2}\right]$.

We begin this section with some general background material enabling us to define the main notions of this article. Assuming for a while that the operator $T$ is injective, we see that for each nonzero $h$ in $\mathcal{H}$, the sequence $\left\{\left\|T^{n+1} h\right\| /\left\|T^{n} h\right\|\right\}_{n=0}^{\infty}$ is bounded above by $\|T\|$. We define $W_{h}$ to be the weighted shift on $l_{+}^{2}$ with this sequence of weights, relative to the standard orthonormal basis $\left\{e_{0}, e_{1}, \ldots\right\}$ of $l_{+}^{2}$. It follows from [9] that each such $W_{h}$ is subnormal and $\left\|W_{h}\right\| \leq\|T\|$. Moreover, by (2.1) for each nonzero $h$ in $\mathcal{H}$, the following equalities hold:

$$
\left\|W_{h}^{n} e_{0}\right\|^{2}=\frac{1}{\|h\|^{2}}\left\|T^{n} h\right\|^{2}=\int t^{n} d \mu_{h}(t), \quad n \geq 0 .
$$

We now briefly review the basic concepts from [3]: given a bounded subnormal (injective) weighted shift $S$ on $l_{+}^{2}$ relative to the orthonormal basis $\left\{e_{0}, e_{1}, \ldots\right\}$ defined by $S e_{n}=\alpha_{n} e_{n+1}$, we say that $S$ has a (subnormal) backward extension if for some scalar $\alpha_{-1} \neq 0$ the weighted shift with weight sequence $\left\{\alpha_{-1}, \alpha_{0}, \alpha_{1}, \ldots\right\}$ is subnormal. R. Curto completely solved the problem of existence of backward extensions.

Theorem 2.1 ([3, Prop. 8]). Let $S$ be a bounded subnormal weighted shift with weight sequence $\left\{\alpha_{0}, \alpha_{1}, \ldots\right\}$ and with associated probability Borel measure $\mu$ on $[0, \infty)$, i.e.

$$
\left\|S^{n} e_{0}\right\|^{2}=\int t^{n} d \mu(t), \quad n \geq 0 .
$$


Then $S$ has a backward extension if and only if 1

$$
\int \frac{1}{t} d \mu(t)<\infty
$$

According to (2.2), the measure $\mu_{h}$ is associated to the weighted shift $W_{h}$. Hence, by Theorem 2.1, the weighted shift $W_{h}$ has a backward extension if and only if $1 / t \in L^{1}\left(\mu_{h}\right)$. This justifies the definitions that follow. From here on, $T$ is not assumed to be injective.

Definition 2.2. A nonzero vector $h \in \mathcal{H}$ is called a one-step backward extension vector for $T$ if $1 / t \in L^{1}\left(\mu_{h}\right)$, where $\mu_{h}$ is as in (2.1). We adhere to the convention that the zero vector is a one-step backward extension vector for $T$. We write $\mathcal{E}_{T}$ for the set of all one-step backward extension vectors for $T$. If $\mathcal{E}_{T}=\mathcal{H}$, then we say that $T$ has one-step full backward extension.

Definition 2.3. Given an integer $k \geq 1$, we say that $T$ has $k$-step full backward extension if $1 / t^{k} \in L^{1}\left(\mu_{h}\right)$ for all nonzero vectors $h \in \mathcal{H}$. If this holds for all integers $k \geq 1$, then we say that $T$ has $\infty$-step full backward extension.

Notice that if $T$ has $k$-step full backward extension and $j$ is an integer such that $1 \leq j \leq k$, then $T$ has $j$-step full backward extension.

Let $N$ be a minimal normal extension of $T$ acting on a Hilbert space $\mathcal{K} \supset \mathcal{H}$ (i.e., $\mathcal{K}$ is the smallest closed linear subspace of $\mathcal{K}$ containing $\mathcal{H}$ and reducing $N$ ), and let $E$ be the spectral measure of $N$. Since $\|N\|=\|T\|$, the closed support of $E$ is contained in the closed disc $\{z \in \mathbb{C}:|z| \leq\|T\|\}$ (cf. [2]). Recall that (cf. [2]) $N$ is a minimal normal extension of $T$ if and only if

$$
\mathcal{K}=\bigvee\left\{N^{* n} h: h \in \mathcal{H}, n \geq 0\right\},
$$

which in turn is equivalent to

$$
\mathcal{K}=\bigvee\{E(\sigma) h: h \in \mathcal{H}, \sigma \text { is a Borel subset of } \mathbb{C}\} .
$$

Set $E_{f}(\cdot)=\langle E(\cdot) f, f\rangle$ for $f \in \mathcal{K}$ and $\rho(z)=|z|^{2}$ for $z \in \mathbb{C}$. It is well known that a vector $f \in \mathcal{K}$ belongs to the range of the normal operator $N$ if and only if $\int \frac{1}{\rho} d E_{f}<\infty$ (with the convention $\frac{1}{0}=\infty$ ). Since for any integer $k \geq 1, N^{k}$ is normal and $E \circ \xi_{k}^{-1}$ is the spectral measure of $N^{k}$, where $\xi_{k}(z)=z^{k}$ for $z \in \mathbb{C}$, we get

Proposition 2.4. For every integer $k \geq 1$ and for every vector $f \in \mathcal{K}, f$ belongs to the range of $N^{k}$ if and only if $\int \frac{1}{\rho^{k}} d E_{f}<\infty$.

The next result relates the measure $\mu_{h}$ appearing in (2.1) to $E_{h}$ as well as describes the ranges of powers of $N$ in terms of $\mu_{h}$.

Lemma 2.5. Let $h$ be a nonzero vector in $\mathcal{H}$. Then

$$
\mu_{h}=\frac{1}{\|h\|^{2}} E_{h} \circ \rho^{-1} .
$$

Moreover, for every integer $k \geq 1$, the following conditions are equivalent:

(i) $1 / t^{k} \in L^{1}\left(\mu_{h}\right)$,

(ii) $1 / z^{k} \in L^{2}\left(E_{h}\right)$,

(iii) $h \in N^{k}(\mathcal{K})$.

\footnotetext{
${ }^{1}$ In the formula (2.3), we adhere to the convention that $\frac{1}{0}=\infty$. In particular, $\int \frac{1}{t} d \mu(t)<\infty$ implies $\mu(\{0\})=0$.
} 
Proof. It follows from (2.1) that

$$
\|h\|^{2} \int t^{n} d \mu_{h}(t)=\left\|T^{n} h\right\|^{2}=\left\|N^{n} h\right\|^{2}=\int \rho^{n} d E_{h}=\int t^{n} d E_{h} \circ \rho^{-1}(t), \quad n \geq 0,
$$

which, by the uniqueness of representing measure, implies (2.6). By virtue of (2.6), we have

$$
\int \frac{1}{t^{k}} d \mu_{h}(t)=\frac{1}{\|h\|^{2}} \int \frac{1}{t^{k}} d E_{h} \circ \rho^{-1}(t)=\frac{1}{\|h\|^{2}} \int \frac{1}{|z|^{2 k}} d E_{h}(z) .
$$

This, when combined with Proposition 2.4, completes the proof.

According to Lemma 2.5, the set $\mathcal{E}_{T}$ coincides with $\mathcal{H} \cap N(\mathcal{K})$.

We are now in a position to formulate necessary and sufficient conditions for $T$ to have $k$-step full backward extension.

Theorem 2.6. If $k \geq 1$ is an integer, then the following conditions are equivalent:

(i) $T$ has $k$-step full backward extension,

(ii) $\mathcal{H}=\mathcal{H} \cap N^{k}(\mathcal{K})$,

(iii) the range of $T^{k}$ is closed and the kernel of $T^{* k}$ is contained in the range of $N^{k}$

(iv) for every $h \in \mathcal{H}$, there exists a positive real constant $c_{h}$ such that

$$
\left|\left(\sum_{i=0}^{n} T^{* i} h_{i}, h\right)\right|^{2} \leq c_{h} \sum_{i, j=0}^{n}\left(T^{j+k} h_{i}, T^{i+k} h_{j}\right), \quad h_{0}, \ldots, h_{n} \in \mathcal{H}, n \geq 0 .
$$

Moreover, if $T$ has $k$-step full backward extension, then both $T$ and $N$ are injective and for every integer $1 \leq j \leq k$, the range of $T^{j}$ is closed and the kernel of $T^{* j}$ is contained in the range of $N^{j}$.

Proof. The equivalence (i) $\Leftrightarrow$ (ii) is a direct consequence of Lemma 2.5. If $T$ has $k$-step full backward extension, then $\mu_{h}(\{0\})=0$ for every nonzero $h \in \mathcal{H}$. Therefore, by (2.6), we have $E(\{0\}) \mathcal{H}=\{0\}$. This in turn leads to $E(\{0\}) E(\sigma) \mathcal{H}=$ $E(\sigma) E(\{0\}) \mathcal{H}=\{0\}$ for every Borel subset $\sigma$ of $\mathbb{C}$. Hence, according to (2.5), $E(\{0\})=0$. This in turn implies that $N$ (and consequently $T$ ) is injective.

(ii) $\Rightarrow$ (iii) It follows from the previous paragraph that $N$ is injective. Thus $\mathcal{H}$ is contained in the domain of $\left(N^{k}\right)^{-1}$ and, in particular, the kernel of $T^{* k}$ is contained in the range of $N^{k}$. Since the operator

$$
\left(N^{k}\right)^{-1}=\int \frac{1}{z^{k}} d E(z)
$$

is closed, so is its restriction to $\mathcal{H}$. Consequently, by the closed graph theorem, there exists $c_{k}>0$ such that $\left\|N^{-k} f\right\| \leq c_{k}\|f\|$ for $f \in \mathcal{H}$. Since $N^{k} h=T^{k} h \in \mathcal{H}$ for $h \in \mathcal{H}$, we get

$$
\|h\|=\left\|N^{-k} T^{k} h\right\| \leq c_{k}\left\|T^{k} h\right\|, \quad h \in \mathcal{H} .
$$

This in turn implies that the range of $T^{k}$ is closed.

(iii) $\Rightarrow$ (ii) Denoting by $\operatorname{ker} T^{* k}$ the kernel of $T^{* k}$, we obtain

$$
\mathcal{H}=T^{k}(\mathcal{H}) \oplus \operatorname{ker} T^{* k}=N^{k}(\mathcal{H}) \oplus \operatorname{ker} T^{* k} \subset N^{k}(\mathcal{K}) .
$$


(ii) $\Leftrightarrow$ (iv) By virtue of the Sebestyén criterion (cf. [11, Th. 1]), $\mathcal{H} \subset N^{k}(\mathcal{K})$ if and only if for every $h \in \mathcal{H}$, there exists $c_{h}>0$ such that

$$
|(g, h)|^{2} \leq c_{h}\left\|N^{* k} g\right\|^{2}, \quad g \in \mathcal{K} .
$$

By (2.4), the inequality (2.8) is equivalent to the following one:

$$
\left|\left(\sum_{i=0}^{n} N^{* i} h_{i}, h\right)\right|^{2} \leq c_{h}\left\|N^{* k}\left(\sum_{i=0}^{n} N^{* i} h_{i}\right)\right\|^{2}, \quad h_{0}, \ldots, h_{n} \in \mathcal{H}, n \geq 0 .
$$

One can now check that (2.9) coincides with (2.7).

The remaining part of the conclusion can be easily deduced from what we have proved above, because if $T$ has $k$-step full backward extension and $1 \leq j \leq k$, then $T$ has $j$-step full backward extension.

Remark 2.7. Referring to Theorem 2.6, notice that if $S$ is an injective bounded linear operator on $\mathcal{H}$ with closed range, then the range of $S^{k}$ is closed for all $k \geq 1$. Indeed, by the open mapping theorem, there exists $c>0$ such that $\|S h\| \geq c\|h\|$ for all $h \in \mathcal{H}$. Hence an induction argument on $k$ shows that $\left\|S^{k} h\right\| \geq c^{k}\|h\|$ for all $h \in \mathcal{H}$ and $k \geq 1$. This in turn implies that the range of $S^{k}$ is closed for all $k \geq 1$.

Corollary 2.8. Suppose $T$ has one-step full backward extension and dense range. Then $T$ is bijective, and it has $\infty$-step full backward extension.

Proof. By Theorem 2.6 and Remark 2.7, $T^{k}$ has closed and dense range. Hence $\mathcal{H}=T^{k}(\mathcal{H})$, which yields $\operatorname{ker} T^{* k}=\{0\}$. Applying Theorem 2.6 completes the proof.

We end this section with orthogonal sums of subnormal operators.

Proposition 2.9. If $T=\bigoplus_{j=1}^{n} T_{j}$ is a finite orthogonal sum of bounded subnormal operators and $k \in\{1,2, \ldots\} \cup\{\infty\}$, then $T$ has $k$-step full backward extension if and only if each $T_{j}$ does as well, $1 \leq j \leq n$.

Proof. It is sufficient to consider the case $k<\infty$. If $N_{j}$ is a minimal normal extension of $T_{j}$, then $N=\bigoplus_{j=1}^{n} N_{j}$ is a minimal normal extension of $T$ and

$$
\mathcal{H} \cap N^{k}(\mathcal{K})=\bigoplus_{j=1}^{n} \mathcal{H}_{j} \cap \bigoplus_{j=1}^{n} N_{j}^{k}\left(\mathcal{K}_{j}\right)=\bigoplus_{j=1}^{n}\left[\mathcal{H}_{j} \cap N_{j}^{k}\left(\mathcal{K}_{j}\right)\right],
$$

which by Theorem 2.6 completes the proof.

The following example shows that Proposition 2.9 is no longer true for infinite orthogonal sums.

Example 2.10. For each $j \geq 1$, let $T_{j}$ be an invertible bounded normal operator on a Hilbert space $\mathcal{H}_{j}$ such that $\left\|T_{j}\right\| \leq 1$ and $\left\|T_{j}^{-1}\right\|=j$ (this can be done even in one-dimensional Hilbert space $\mathcal{H}_{j}$ ). Then by Lemma 3.1 each $T_{j}$ has onestep full backward extension, while the bounded normal operator $T=\bigoplus_{j=1}^{\infty} T_{j}$ 
does not have one-step full backward extension. Indeed, otherwise once more by Lemma 3.1 there exists a positive constant $c$ such that $\|T h\| \geq c\|h\|$ for all $h \in \mathcal{H}$. Consequently, $\left\|T_{j} h\right\| \geq c\|h\|$ for all $h \in \mathcal{H}_{j}$ and $j \geq 1$. This in turn implies that $\left\|T_{j}^{-1}\right\| \leq \frac{1}{c}$ for all $j \geq 1$, which contradicts our assumption.

\section{Quasinormal operators}

We now examine a class of operators between the normal and subnormal classes. Recall that a bounded linear operator $T$ on a Hilbert space $\mathcal{H}$ is quasinormal if $T$ commutes with $T^{*} T$ (cf. [2]). Every normal operator is quasinormal, and every quasinormal operator is subnormal. In infinite-dimensional Hilbert spaces these set containments are proper.

Let us begin with the case of a normal operator.

Lemma 3.1. If $T$ is a bounded normal operator on $\mathcal{H}$ and $k \geq 1$ is an integer, then the following conditions are equivalent:

(i) T has one-step full backward extension,

(ii) $T$ has $k$-step full backward extension,

(iii) $T$ has $\infty$-step full backward extension,

(iv) $T$ is injective and the range of $T$ is closed,

(v) $T$ is bijective and $T^{-1}$ is a bounded operator on $\mathcal{H}$.

Proof. The implication (i) $\Rightarrow$ (iv) is a consequence of Theorem 2.6. The implication (iv) $\Rightarrow(\mathrm{v})$ can be deduced from the open mapping theorem and the fact that the kernels of $T$ and $T^{*}$ coincide.

(v) $\Rightarrow$ (iii) Since the minimal normal extension $N$ of $T$ equals $T$, we get $\mathcal{H}=$ $N^{k}(\mathcal{K})$ for all $k \geq 1$. Thus, by Theorem 2.6, $T$ has $\infty$-step full backward extension.

The implications (iii) $\Rightarrow$ (ii) $\Rightarrow$ (i) are clear.

The next lemma deals with the case of a pure quasinormal operator. A bounded quasinormal operator $T$ on $\mathcal{H}$ is said to be pure, if the only closed linear subspace of $\mathcal{H}$ reducing $T$ to a normal operator is $\{0\}$. In [1], A. Brown shows that if $T$ is a bounded pure quasinormal operator on $\mathcal{H}$, then there exists an orthogonal decomposition of $\mathcal{H}$ as $\mathcal{M} \oplus \mathcal{M} \oplus \cdots$, where $\mathcal{M}$ is a closed linear subspace of $\mathcal{H}$, and a corresponding matrix representation of $T$ as $A \otimes V$, where $V$ is the unilateral shift of multiplicity one and $A$ is a bounded injective positive operator on $\mathcal{M}$.

Lemma 3.2. Let $T$ be a bounded pure quasinormal operator on $\mathcal{H}$, and let $k \geq 1$ be an integer. If $A \otimes V$ is a matrix representation of $T$, where $A$ is a bounded injective positive operator on $\mathcal{M}$, then the following conditions are equivalent:

(i) $T$ has one-step full backward extension,

(ii) $T$ has $k$-step full backward extension,

(iii) $T$ has $\infty$-step full backward extension,

(iv) the range of $T$ is closed,

(v) $A^{-1}$ is a bounded operator on $\mathcal{M}$.

Proof. The implication (i) $\Rightarrow$ (iv) is a consequence of Theorem 2.6.

(iv) $\Rightarrow$ (v) We first show that the range of $A$ is closed. Take $g$ in the closure of $A(\mathcal{M})$. Then there exists a sequence $\left\{h_{n}\right\}_{n=1}^{\infty} \subset \mathcal{M}$ such that $A h_{n} \rightarrow g$ as $n \rightarrow \infty$. Hence

$$
T\left(h_{n} \oplus 0 \oplus \cdots\right)=0 \oplus A h_{n} \oplus 0 \oplus \cdots \rightarrow 0 \oplus g \oplus 0 \oplus \cdots \quad \text { as } n \rightarrow \infty .
$$


Since the range of $T$ is closed, there exists $f_{0} \oplus f_{1} \oplus \cdots$ in $\mathcal{H}$ with all $f_{i}$ in $\mathcal{M}$ such that

$$
0 \oplus g \oplus 0 \oplus \cdots=T\left(f_{0} \oplus f_{1} \oplus \cdots\right)=0 \oplus A\left(f_{0}\right) \oplus A\left(f_{1}\right) \oplus \cdots .
$$

Consequently, we get $g=A\left(f_{0}\right)$, which proves our assertion. The closedness of the range of $A$ and the selfadjointness of $A$ imply $A(\mathcal{M})=\mathcal{M}$. This in turn, by the open mapping theorem, guarantees the boundedness of $A^{-1}$.

(v) $\Rightarrow$ (iii) The minimal normal extension $N$ of $T$ has the matrix representation $A \otimes B$, where $B$ is the bilateral shift of multiplicity one, i.e., $N$ is a bilateral operator weighted shift with constant weights $A$. If $f=f_{0} \oplus f_{1} \oplus \cdots \in \mathcal{H}$ with all $f_{i}$ in $\mathcal{M}$, then for every integer $k \geq 0$, the vector $g^{(k)}$ defined by

$$
g^{(k)}=\cdots \oplus \underbrace{0}_{(-k-1) \text {-th }} \oplus \underbrace{A^{-k} f_{0}}_{(-k+0) \text {-th }} \oplus \underbrace{A^{-k} f_{1}}_{(-k+1) \text {-th }} \oplus \underbrace{A^{-k} f_{2}}_{(-k+2) \text {-th }} \oplus \cdots
$$

belongs to $\mathcal{K}$ (because $A^{-1}$ is bounded) and $f=N^{k} g^{(k)} \in N^{k}(\mathcal{K})$. Thus $\mathcal{H}=$ $\mathcal{H} \cap N^{k}(\mathcal{K})$ for all $k \geq 1$. By Theorem 2.6, $T$ has $\infty$-step full backward extension.

The implications (iii) $\Rightarrow$ (ii) $\Rightarrow$ (i) are straightforward.

Given a quasinormal operator $T$ on $\mathcal{H}$, we denote by $T_{\text {nor }}$ and $T_{\mathrm{p}}$ the normal and the pure part of $T$, respectively. The operators $T_{\text {nor }}$ and $T_{\mathrm{p}}$ act on spaces $\mathcal{H}_{\text {nor }}$ and $\mathcal{H}_{\mathrm{p}}$, respectively. Since $T_{\mathrm{p}}$ is a pure quasinormal operator, it has a matrix representation of the form $A \otimes V$, where $A$ is a bounded injective positive operator or $\mathcal{M}$.

Theorem 3.3. Let $T$ be a bounded quasinormal operator on $\mathcal{H}$, and let $k \geq 1$ be an integer. If $A \otimes V$ is a matrix representation of $T_{\mathrm{p}}$, where $A$ is a bounded injective positive operator on $\mathcal{M}$, then the following conditions are equivalent:

(i) T has one-step full backward extension,

(ii) $T$ has $k$-step full backward extension,

(iii) $T$ has $\infty$-step full backward extension,

(iv) $T$ is injective and the range of $T$ is closed,

(v) $T_{\text {nor }}$ is bijective, $T_{\text {nor }}^{-1}$ is a bounded operator on $\mathcal{H}_{\text {nor }}$ and $A^{-1}$ is a bounded operator on $\mathcal{M}$.

Proof. Apply Proposition 2.9 and Lemmas 3.1 and 3.2.

By virtue of part (v) of Theorem 3.3, there exist injective normal operators as well as pure quasinormal operators that do not have one-step full backward extensions.

Example 3.4. Let $(X, \mathcal{U}, \beta)$ be an arbitrary measure space with a positive measure $\beta$. For a $\beta$-essentially bounded positive $\mathcal{U}$-measurable function $a$ on $X$ we define the bounded injective positive operator $A$ on $L^{2}(X, \beta)$ by $A h(x)=a(x) h(x)$ for $h \in L^{2}(X, \beta)$. Let $T$ be the bounded quasinormal operator on $l_{+}^{2}\left(L^{2}(X, \beta)\right)$ of the

\footnotetext{
${ }^{2}$ The case $\mathcal{M}=\{0\}$ is not excluded.
} 
form $A \otimes V$. If $h=\bigoplus_{i=0}^{\infty} h_{i} \in l_{+}^{2}\left(L^{2}(X, \beta)\right)$ is nonzero, then by (2.1) we have

$$
\begin{aligned}
\|h\|^{2} \int t^{n} d \mu_{h}(t) & =\left\|T^{n} h\right\|^{2}=\sum_{i=0}^{\infty}\left\|A^{n} h_{i}\right\|_{L^{2}(X, \beta)}^{2} \\
& =\int_{X} a^{2 n} \sum_{i=0}^{\infty}\left|h_{i}\right|^{2} d \beta \\
& =\int_{X} a^{2 n} d \alpha_{h} \quad\left(\text { where } d \alpha_{h}=\sum_{i=0}^{\infty}\left|h_{i}\right|^{2} d \beta\right) \\
& =\int t^{n} d \alpha_{h} \circ \varphi^{-1}(t) \quad\left(\text { where } \varphi=a^{2}\right), \quad n \geq 0 .
\end{aligned}
$$

Hence $\mu_{h}=\frac{1}{\|h\|^{2}} \alpha_{h} \circ \varphi^{-1}$.

Now we have two possibilities. If the function $\frac{1}{a}$ is $\beta$-essentially bounded, then $A^{-1}$ is a bounded operator on $L^{2}(X, \beta)$, and consequently by Lemma 3.2 the operator $T$ has $\infty$-step full backward extension. On the other hand, if the function $\frac{1}{a}$ is not $\beta$-essentially bounded, then $A^{-1}$ is not bounded, and consequently by Lemma 3.2 the operator $T$ does not have one-step full backward extension. By the way, according to Lemma 3.1, the operator $A$ does not have one-step full backward extension.

\section{Cyclic subnormal operators}

Suppose that $T$ is a cyclic subnormal operator on a Hilbert space $\mathcal{H}$, and let $N$ be its minimal normal extension. For brevity, we assume that $\|T\|=1$ throughout this section. Then $T$ is unitarily equivalent to a multiplication operator $S_{\nu}$ on $H^{2}(\nu)$ with normal extension $N_{\nu}$ on $L^{2}(\nu)$, where the subscript $\nu$ indicates multiplication by the independent variable $z$ on $H^{2}(\nu)$ or $L^{2}(\nu)$. For nonzero $h$ in $H^{2}(\nu)$, let $d \nu_{h}=|h|^{2} d \nu$. Also, let $\rho(z)=|z|^{2}$. Then

$$
\left\|S_{\nu}^{n} h\right\|^{2}=\int_{\mathbb{D}}\left|z^{n} h(z)\right|^{2} d \nu(z)=\int_{\mathbb{D}} \rho^{n} d \nu_{h}=\int_{0}^{1} t^{n} d \nu_{h} \circ \rho^{-1}(t), \quad n \geq 0 .
$$

Applying (2.1) to $T=S_{\nu}$, we get

$$
\mu_{h}=\frac{1}{\|h\|^{2}} \cdot \nu_{h} \circ \rho^{-1}
$$

This in turn implies that for every integer $k \geq 1$,

$$
\|h\|^{2} \int_{0}^{1} \frac{1}{t^{k}} d \mu_{h}(t)=\int_{0}^{1} \frac{1}{t^{k}} d \nu_{h} \circ \rho^{-1}(t)=\int_{\mathbb{D}} \frac{1}{\rho^{k}} d \nu_{h}=\int_{\mathbb{D}}\left|\frac{h(z)}{z^{k}}\right|^{2} d \nu(z) .
$$

Lemma 4.1. For every integer $k \geq 1$, the following are equivalent:

(a) $S_{\nu}$ has $k$-step full backward extension,

(b) $\int_{\mathbb{D}}\left|h(z) / z^{k}\right|^{2} d \nu(z)<\infty$ for all $h \in H^{2}(\nu)$ (equivalently: $1 / z^{k} \cdot H^{2}(\nu) \subset$ $\left.L^{2}(\nu)\right)$,

(c) there exists $c>0$ such that for all $h \in H^{2}(\nu)$ (equivalently: for all polynomials $h$ ),

$$
\int_{\mathbb{D}}\left|h(z) / z^{k}\right|^{2} d \nu(z) \leq c \int_{\mathbb{D}}|h(z)|^{2} d \nu(z)
$$


Proof. That (a) is equivalent to (b) follows from (4.2). Notice that the operator $\Theta_{k}: H^{2}(\nu) \supset D\left(\Theta_{k}\right) \longrightarrow L^{2}(\nu)$ defined on the domain $D\left(\Theta_{k}\right)=\left\{h \in H^{2}(\nu)\right.$ : $\left.1 / z^{k} \cdot h \in L^{2}(\nu)\right\}$ via $\Theta_{k}(h)=1 / z^{k} \cdot h, h \in D\left(\Theta_{k}\right)$, is closed. If (b) holds, then $D\left(\Theta_{k}\right)=H^{2}(\nu)$ and consequently, by the closed graph theorem, (4.3) holds for all $h \in H^{2}(\nu)$. If (4.3) holds for all polynomials $h$, then $\mathcal{P} \subset D\left(\Theta_{k}\right)$ and $\left\|\left.\Theta_{k}\right|_{\mathcal{P}}\right\| \leq \sqrt{c}$, where $\mathcal{P}$ is the set of all polynomials on $\mathbb{D}$. Hence $D\left(\Theta_{k}\right)=H^{2}(\nu)$ and $\left\|\Theta_{k}\right\| \leq \sqrt{c}$, which means that (4.3) holds for all $h \in H^{2}(\nu)$.

We write $R_{k}^{2}(\nu):=H^{2}(\nu) \vee\left\{1 / z, \ldots, 1 / z^{k}\right\}$ and $R^{2}(\nu):=H^{2}(\nu) \vee\left\{1 / z^{k}: k \geq 1\right\}$. These definitions are restricted to the case that the appropriate $1 / z^{k}$ terms are in $L^{2}(\nu)$, and the span refers to the $L^{2}(\nu)$-closed linear span. If $1 / z^{k} \in L^{2}(\nu)$ (hence $1 / z^{i} \in L^{2}(\nu)$ for $\left.i=1, \ldots, k\right)$, then $T^{(k)}:=\left.N_{\nu}\right|_{R_{k}^{2}(\nu)}$ is a well-defined cyclic subnormal operator (of norm one) with the cyclic vector $1 / z^{k}$. Since $N_{\nu}$ is a minimal normal extension of $S_{\nu}$, we see that $N_{\nu}$ is a minimal normal extension of $T^{(k)}$. Denote by $\nu^{(k)}$ the measure associated with $T^{(k)}$ and by $\mu_{h}^{(k)}$ the corresponding measure on $[0,1]$ for nonzero $h \in H^{2}\left(\nu^{(k)}\right)$.

Proposition 4.2. Let $S_{\nu}$ be the cyclic subnormal operator on $H^{2}(\nu)$. Then

(a) $\frac{1}{\|1\|_{L^{2}(\nu)}^{2}} \nu \circ \rho^{-1}=\mu_{1}$.

Let $k \geq 1$ be an integer such that $1 / z^{k} \in L^{2}(\nu)$. Then

(b) $d \nu^{(k)}(z)=\frac{1}{|z|^{2}} d \nu^{(k-1)}(z)$ with $\nu^{(0)}=\nu$,

(c) $d \nu^{(k)}(z)=\frac{1}{|z|^{2 k}} d \nu(z)$,

(d) $d \nu^{(k)} \circ \rho^{-1}(t)=\|1\|_{L^{2}(\nu)}^{2} \frac{1}{t^{k}} d \mu_{1}(t)$,

(e) $d \mu_{1}^{(k)}(t)=\frac{\|1\|_{L^{2}(\nu)}^{2}}{\left\|1 / z^{k}\right\|_{L^{2}(\nu)}^{2}} \frac{1}{t^{k}} d \mu_{1}(t)$,

(f) $\nu^{(k)} \ll \nu \ll \nu^{(k)}, H^{2}\left(\nu^{(k)}\right)=z^{k} \cdot R_{k}^{2}(\nu)$ and $H^{2}\left(\nu^{(k)}\right) \subset H^{2}(\nu)$.

Proof. (a) Use (4.1).

(c) Let $U^{(k)}: R_{k}^{2}(\nu) \rightarrow H^{2}\left(\nu^{(k)}\right)$ be the unitary operator such that $U^{(k)} T^{(k)}=$ $S_{\nu^{(k)}} U^{(k)}$ and $U^{(k)}\left(1 / z^{k}\right)=1$ (the unitary operator $U^{(k)}$ gives us the $L^{2}\left(\nu^{(k)}\right)$-model $S_{\nu^{(k)}}$ of $\left.T^{(k)}\right)$. Then $U^{(k)}\left(z^{n} / z^{k}\right)=U^{(k)}\left(T^{(k)}\right)^{n}\left(1 / z^{k}\right)=S_{\nu^{(k)}}^{n} U^{(k)}\left(1 / z^{k}\right)=z^{n}$ for all $n \geq 0$. Hence

$$
\begin{aligned}
\int_{\mathbb{D}} z^{m} \bar{z}^{n} d \nu^{(k)}(z) & =\left(U^{(k)}\left(z^{m} / z^{k}\right)\right),\left(U^{(k)}\left(z^{n} / z^{k}\right)\right)_{L^{2}\left(\nu^{(k)}\right)} \\
& =\int_{\mathbb{D}} z^{m} \bar{z}^{n} \frac{1}{|z|^{2 k}} d \nu(z), \quad m, n \geq 0 .
\end{aligned}
$$

Since bounded complex moment sequences are determinate, the above equalities imply (c). (c) in turn gives us (b).

(d) By (c), for every Borel subset $\sigma$ of $[0,1]$, we have

$$
\nu^{(k)} \circ \rho^{-1}(\sigma)=\int_{\mathbb{D}} \frac{1}{\rho^{k}} \chi_{\rho^{-1}(\sigma)} d \nu=\int_{\mathbb{D}} \frac{1}{\rho^{k}} \chi_{\sigma} \circ \rho d \nu=\int_{\sigma} \frac{1}{t^{k}} d \nu \circ \rho^{-1}(t) .
$$

This and (a) imply (d).

(e) follows directly from (a) and (d).

(f) That $\nu^{(k)}$ and $\nu$ are mutually absolutely continuous follows from (c) and $\nu(\{0\})=0$. By $(\mathrm{c})$, the mapping $\Phi_{k}: H^{2}\left(\nu^{(k)}\right) \longrightarrow L^{2}(\nu)$ given by $\Phi_{k}(h)=$ $1 / z^{k} \cdot h$ is an isometry. Hence $\Phi_{k}\left(H^{2}\left(\nu^{(k)}\right)\right)=\Phi_{k}\left(\bigvee_{n=0}^{\infty} z^{n}\right)=\bigvee_{n=0}^{\infty} \Phi_{k}\left(z^{n}\right)=$ 
$R_{k}^{2}(\nu)$. Consequently, $H^{2}\left(\nu^{(k)}\right)=z^{k} \cdot R_{k}^{2}(\nu)$. This and the continuity of $N_{\nu}^{k}$ lead to $H^{2}\left(\nu^{(k)}\right)=N_{\nu}^{k}\left(R_{k}^{2}(\nu)\right) \subset H^{2}(\nu)$.

By Lemma 4.1, if $S_{\nu}$ has $k$-step full backward extension, then $1 / z^{k} \in L^{2}(\nu)$. The question then arises as to what condition in addition to $1 / z^{k} \in L^{2}(\nu)$ is necessary to guarantee that $S_{\nu}$ has $k$-step full backward extension. The prototype for the solution may be taken to be a subnormal weighted shift, which always has closed range.

Theorem 4.3. Let $k \geq 1$ be an integer.

(a) $S_{\nu}$ has $k$-step full backward extension if and only if the range of $S_{\nu}^{k}$ is closed and $1 / z^{k} \in L^{2}(\nu)$ (the latter is equivalent to $1 / t^{k} \in L^{1}\left(\mu_{1}\right)$ ).

(b) $S_{\nu}$ has $k$-step full backward extension if and only if $1 / z^{k} \in L^{2}(\nu)$ and $H^{2}\left(\nu^{(k)}\right)=H^{2}(\nu)$.

(c) If $S_{\nu}$ has $k$-step full backward extension $(k \geq 2)$, then for each $i=1, \ldots, k-1$, the operator $S_{\nu^{(i)}}$ has $(k-i)$-step full backward extension.

Proof. (a) The "only if" part of (a) follows from Lemma 4.1 and Theorem 2.6.

Assume now that the range of $S_{\nu}^{k}$ is closed. Since

$$
\bigvee_{j=k}^{\infty} z^{j}=\bigvee_{j=k}^{\infty} S_{\nu}^{k}\left(z^{j-k}\right) \subset S_{\nu}^{k}\left(H^{2}(\nu)\right)=S_{\nu}^{k}\left(\bigvee_{n=0}^{\infty} z^{n}\right) \subset \bigvee_{n=0}^{\infty} S_{\nu}^{k}\left(z^{n}\right)=\bigvee_{j=k}^{\infty} z^{j}
$$

we get

$$
S_{\nu}^{k}\left(H^{2}(\nu)\right)=\bigvee_{j=k}^{\infty} z^{j}
$$

Next, we have

$$
H^{2}(\nu)=\bigvee\left\{1, z, \ldots, z^{k-1}\right\}+\bigvee_{j=k}^{\infty} z^{j}
$$

because the right side of (4.5) is closed (as the algebraic sum of a closed linear space and a finite-dimensional one) and dense in $H^{2}(\nu)$. Assume, additionally, that $1 / z^{k} \in L^{2}(\nu)$. If $h \in H^{2}(\nu)$, then by (4.4) and (4.5), there exist $\alpha_{0}, \alpha_{1}, \ldots, \alpha_{k-1} \in \mathbb{C}$ and $g \in H^{2}(\nu)$ such that $h=\alpha_{0}+\alpha_{1} z+\cdots+\alpha_{k-1} z^{k-1}+z^{k} g$. Hence $h / z^{k} \in L^{2}(\nu)$ and $h=N_{\nu}^{k}\left(h / z^{k}\right) \in N_{\nu}^{k}\left(L^{2}(\nu)\right)$. This shows that $H^{2}(\nu) \subset N_{\nu}^{k}\left(L^{2}(\nu)\right)$. Applying Theorem 2.6 completes the proof of (a).

(b) By Lemma 4.1, we can assume that $1 / z^{k} \in L^{2}(\nu)$. If $H^{2}\left(\nu^{(k)}\right)=H^{2}(\nu)$, then by part (f) of Proposition 4.2, the operator $\Psi_{k}: R_{k}^{2}(\nu) \longrightarrow H^{2}(\nu)$ given by $\Psi_{k}(h)=z^{k} \cdot h, h \in R_{k}^{2}(\nu)$, is bijective. Hence, if $h \in H^{2}(\nu)$, then $1 / z^{k} \cdot h=\Psi_{k}^{-1}(h) \in$ $L^{2}(\nu)$. In view of Lemma 4.1, $S_{\nu}$ has $k$-step full backward extension. Conversely, if $S_{\nu}$ has $k$-step full backward extension, then by Lemma 4.1 the operator $\Theta_{k}$ : $H^{2}(\nu) \longrightarrow L^{2}(\nu)$ defined by $\Theta_{k}(h)=1 / z^{k} \cdot h, h \in H^{2}(\nu)$, is continuous. Thus $\Theta_{k}\left(H^{2}(\nu)\right)=\Theta_{k}\left(\bigvee_{n=0}^{\infty} z^{n}\right) \subset \bigvee_{n=0}^{\infty} \Theta_{k}\left(z^{n}\right)=R_{k}^{2}(\nu)$. Consequently, if $h \in H^{2}(\nu)$, then $g:=1 / z^{k} \cdot h \in R_{k}^{2}(\nu)$, which together with part (f) of Proposition 4.2 gives us $h=z^{k} \cdot g \in z^{k} \cdot R_{k}^{2}(\nu)=H^{2}\left(\nu^{(k)}\right)$. This shows that $H^{2}(\nu) \subset H^{2}\left(\nu^{(k)}\right)$. Applying once more part (f) of Proposition 4.2 completes the proof of (b).

(c) By Lemma 4.1 and part (c) of Proposition 4.2, we have

$$
\int_{\mathbb{D}}\left|\frac{1}{z^{k-i}}\right|^{2} d \nu^{(i)}(z)=\int_{\mathbb{D}}\left|\frac{1}{z^{k}}\right|^{2} d \nu(z)<\infty
$$


This and part (c) of Proposition 4.2 enable us to show that $\left(\nu^{(i)}\right)^{(k-i)}=\nu^{(k)}$. It follows from (b) that $H^{2}\left(\left(\nu^{(i)}\right)^{(k-i)}\right)=H^{2}\left(\nu^{(k)}\right)=H^{2}(\nu)=H^{2}\left(\nu^{(i)}\right)$. This, (4.6) and (b) (applied to $\left.S_{\nu^{(i)}}\right)$ complete the proof of (c).

Corollary 4.4. If $k \geq 1$, then a subnormal weighted shift $T$ on $\mathcal{H}$ (of norm one) has $k$-step full backward extension if and only if $1 / t^{k} \in L^{1}\left(\mu_{e_{0}}\right)$, where $\left\{e_{n}\right\}_{n=0}^{\infty}$ is an orthonormal basis of $\mathcal{H}$ with respect to which the weighted shift is defined.

Proof. Since each (injective) subnormal weighted shift has closed range (hence all its powers have closed ranges, cf. Remark 2.7), the conclusion follows from Theorem 4.3 (the cyclic vector $e_{0}$ of $T$ is represented in the model space $H^{2}(\nu)$ by the constant function 1).

Example 4.5. Let $k \geq 1$. If $\mu$ is a probability Borel measure on $[0,1]$ such that 1 belongs to the closed support of $\mu, 1 / t^{k} \in L^{1}(\mu)$ and $1 / t^{k+1} \notin L^{1}(\mu)$ (e.g., $\left.d \mu(t)=(k+1) t^{k} d t\right)$, then there is an injective weighted shift $T$ of norm one such that $\mu_{e_{0}}=\mu$, and consequently, by Corollary 4.4, $T$ has $k$-step full backward extension but not $(k+1)$-step full backward extension. On the other hand, if $d \mu(t)=a e^{-1 / t} d t$ (with an appropriate normalizing positive constant $a$ ), then $T$ has $\infty$-step full backward extension.

Proposition 4.6. If $S_{\nu}$ has one-step full backward extension and dense range, then $S_{\nu}$ has $\infty$-step full backward extension, $R^{2}(\nu)=H^{2}(\nu)$ and $H^{2}\left(\nu^{(k)}\right)=H^{2}(\nu)$ for all $k \geq 1$.

Proof. By Corollary 2.8, $H^{2}(\nu)=S_{\nu}^{i} H^{2}(\nu)$, which implies $1=S_{\nu}^{i} g_{i}$ for some $g_{i} \in H^{2}(\nu)$. Thus $1 / z^{i}=g_{i} \in H^{2}(\nu)$, which shows that $R^{2}(\nu)=H^{2}(\nu)$. Applying Corollary 2.8 and part (b) of Theorem 4.3 completes the proof.

Remark 4.7. It was shown in [5] that if the cyclic vector $e_{0}$ admits one-step backward extension for a weighted shift, then the shift has one-step full backward extension. The following example shows that this is not the case for general cyclic subnormal operators.

Example 4.8. Let $d \nu=3 t^{2} d t$ on $[0,1]$. Then $H^{2}(\nu)=L^{2}(\nu)$ and $S_{\nu} f(t)=t f(t)$. Since $1 / t \in L^{1}(\nu)$, we get $1 \in \mathcal{E}_{S_{\nu}}$. Let $f(t)=1 / t$. Then $f \in H^{2}(\nu)$ and $\int_{0}^{1}\left|\frac{f(t)}{t}\right|^{2} d \nu(t)=\infty$. So $f \notin \mathcal{E}_{S_{\nu}}$, and hence $H^{2}(\nu) \neq \mathcal{E}_{S_{\nu}}$ (the latter follows also from Lemma 3.1).

\section{ACKNOWLEDGMENT}

A substantial part of this paper was written while the second author visited Kyungpook National University during the summer of 2001 and was finished while the third author visited the same university during the fall of 2002; they wish to thank the faculty and administration of those units for their warm hospitality. The first author was supported by the Korea Research Foundation Grant (KRF-2001015-DP0010). The third author was supported by the KBN Grant 2 P03A 037 024. 


\section{REFERENCES}

[1] A. Brown, On a class of operators, Proc. Amer. Math. Soc. 4 (1953), 723-728. MR 15:538c

[2] J. Conway, Subnormal operators, Pitman Publ. Co., London, 1981. MR 83i:47030

[3] R. Curto, Quadratically hyponormal weighted shifts, Integral Equations Operator Theory 13 (1990), 49-66. MR 90k:47061

[4] M. Embry, A generalization of the Halmos-Bram criterion for subnormality, Acta Sci. Math. (Szeged) 35 (1973), 61-64. MR 48:6994

[5] T. Hoover, I. Jung, and A. Lambert, Moment sequences and backward extensions of subnormal weighted shifts, J. Austral. Math. Soc. 73 (2002), 27-36. MR 2003e:47056

[6] I. Jung and C. Li, A formula for k-hyponormality of backstep extensions of subnormal weighted shifts, Proc. Amer. Math. Soc. 129 (2001), 2343-2351. MR 2002b:47061

[7] I. Jung and S. Park, Quadratically hyponormal weighted shifts and their examples, Integral Equations and Operator Theory 36 (2000), 480-498. MR 2001i:47051

[8] I. Jung and S. Park, Cubically hyponormal weighted shifts and their examples, J. Math. Anal. Appl. 247 (2000), 557-569. MR 2001h:47047

[9] A. Lambert, Subnormality and weighted shifts, J. London Math. Soc. 14 (1976), 476-480. MR 55:8866

[10] A. Lambert, Subnormal composition operators, Proc. Amer. Math. Soc. 103 (1988), 750-754. MR 89k:47033

[11] Z. Sebestyén, On ranges of adjoint operators in Hilbert space, Acta Sci. Math. (Szeged) 46 (1983), 295-298. MR 85i:47003a

[12] A. Shields, Weighted shift operators and analytic function theory, Topics in Operator Theory, Math. Surveys 13, Amer. Math. Soc., Providence, R.I., 1974. MR 50:14341

[13] J. Stochel, Seminormality of operators from their tensor product, Proc. Amer. Math. Soc. 124 (1996), 135-140. MR 96d:47030

Department of Mathematics, College of Natural Sciences, Kyungpook National UniVERsity, Daegu 702-701, Korea

E-mail address: ibjung@knu.ac.kr

Department of Mathematics, University of North Carolina at Charlotte, UnCC Station, Charlotte, North Carolina 28223

E-mail address: allamber@email.uncc.edu

Instytut Matematyki, Uniwersytet Jagielloński, Ul. Reymonta 4, PL-30059 Kraków, POLAND

E-mail address: stochel@im.uj.edu.pl 